MATEC Web of Conferences 45, 04010 (2016)

DOI: $10.1051 /$ matecconf/20164504010

(c) Owned by the authors, published by EDP Sciences, 2016

\title{
Fuzzy model for Laser Assisted Bending Process
}

\author{
Oliviero Giannini , Stefano Guarino \\ Università degli Studi Niccolò Cusano - via Don Gnocchi 3, 00166 Rome, Italy
}

\begin{abstract}
In the present study, a fuzzy model was developed to predict the residual bending in a conventional metal bending process assisted by a high power diode laser. The study was focused on AA6082T6 aluminium thin sheets. In most dynamic sheet metal forming operations, the highly nonlinear deformation processes cause large amounts of elastic strain energy stored in the formed material. The novel hybrid forming process was thus aimed at inducing the local heating of the mechanically bent workpiece in order to decrease or eliminate the related springback phenomena. In particular, the influence on the extent of springback phenomena of laser process parameters such as source power, scan speed and starting elastic deformation of mechanically bent sheets, was experimentally assessed. Consistent trends in experimental response according to operational parameters were found. Accordingly, 3D process maps of the extent of the springback phenomena according to operational parameters were constructed. The effect of the inherent uncertainties on the predicted residual bending caused by the approximation in the model parameters was evaluated. In particular, a fuzzy-logic based approach was used to describe the model uncertainties and the transformation method was applied to propagate their effect on the residual bending.
\end{abstract}

\section{Introduction}

Bending processes are widely used in various manufacturing domains to produce a large variety of geometrical shape starting from raw shape and components. [1-4]. The main drawback of the bending process is the springback phenomenon. The springback is the result of the energy stored inside the processed workpiece when plastically deformed during the bending process. This energy is released when the constraints are removed at the end of the bending process, leading to an unexpected change in the final shape of the workpiece due to residual elasticity kept inside the bent material [1]. This aspect can cause serious error in the final shape of the workpiece compromising the process performance in terms of lack of the closest shape and dimensional tolerances. Several studies in the literature deal with the analysis of the springback in metal sheet forming and its compensation [5-7].

In recent years, the laser forming process has reached a considerable importance as promising technology in many manufacturing domains, such as automobile, microelectronics and aerospace [1-4]. The laser forming process has many advantages. First, it requires no external forces, so the springback may be negligible. Second, it can shape complex surface producing even very small parts. Moreover, many hard and brittle materials, such as titanium alloy, nickel alloy, ceramics, etc., can be processed. Unfortunately laser forming is a time-consuming process, when compared to traditional technology.
In this paper, a diode laser was used to assist the traditional forming process. The laser focused on the critical areas during the sheet forming induces a localized heating of the component that permits the release of the energy stored during the forming process reducing the springback phenomena. This application of laser in forming process is relatively new and the few studies are available in the literature [6-8]. The problems related to this approach are due to the lack of understanding of leading phenomena involved as well as process simulation and modelling.

The present work proposes an experimental investigation and a fuzzy modeling on laser-assisted bending process of aluminium sheet. The study is focused on the evaluation and control of the springback extent by assisting the forming operations with a selective heating of the bent area by irradiation with a high power diode laser. All the experimental tests were aimed at understanding the basic mechanisms involved in the laser-assisted forming process and to study the operational parameters of the process (laser power, scan speed and imposed inflection). Then a fuzzy methodology is applied to a regression model used to predict the residual bending. This third application of the method allows the complete formalization of the procedure based on fuzzy approach to the process. In particular, this approach allowed:

(i) to evaluate how much a given sample, characterized by a combination of process parameters and the corresponding residual bending level, belonged to the fuzzy set defined by the model; 
(ii) to evaluate the percentage of samples which were described by the model for a given membership level, and thus ;

(iii) to select operational parameters and predict the fidelity of the model at the operational point chosen.

\section{Methodology}

\subsection{The machining process}

Laser-assisted bending process was carried out on specimen of AA6082T6 aluminium alloy with the dimensions of $150 \times 30 \times 1.2 \mathrm{~mm}$. The geometrical tolerances $(<0.05 \mathrm{~mm})$ and uniform starting surface conditions were assured by controls performed with coordinate-measuring machine (Mitutoyo CRISTA APEX C544). An ultrasound cleaning system (SOLTEC model Sonica 2200) was utilized with the aim of having the same cleanliness for all the specimens.

Laser processing was performed using a diode laser source (ROFIN-SINAR, model DL 015) with a wavelength of $940 \pm 10 \mathrm{~nm}$ and an elliptical spot, with dimensions of $1.9 \mathrm{~mm}$ along its major axis and $0.6 \mathrm{~mm}$ along other axis..For protection and isolation purposes an argon flux was flushed on the substrate surface during the laser treatment. The focal distance of the lens was $63 \mathrm{~mm}$, while the working distance was $32 \mathrm{~mm}$.

The aim of this experimentation was to understand the effects of the laser on the elastic properties of the material. All the tests were performed by bending the specimens in the elastic range and treating them with a diode laser. The heating of the material induces a reduction of the elastic energy that the material is able to store and, as a consequence, the reduction of the springback phenomenon $[7,8]$.

The steps of experimental procedure were as follows:

(i) the specimens were cleaned by using ultrasound cleaning;

(ii) the aluminium sheets were placed in a three points bending load system with which it was possible to induce a fixed starting inflexion in the elastic range. Figure 1 depicts a diagram of the load system used in the experimentation;

(iii) the specimens bent in the elastic range were treated with the diode laser along the medium axis (highlighted with the blue arrow in figure 1)

(iv) After the removal of the applied load, the residual inflection of the aluminium sheets was monitored with a Taylor-Hobson profilometer. Ten profiles along the axial direction and at a distance of $0.5 \mathrm{~mm}$ from each other were acquired. The maximum inflection value was measured for all the profiles. The mean of this inflexion values represents the residual inflexion considered. The measure of the straightness of the specimens using the profilometer was taken to assure the same starting conditions before the laser treatment

Table 1 summarizes the experimental plan used. Three operational parameters were considered: laser power (range 500 to $800 \mathrm{~W}$ ), laser scan speed (range 4 to $8 \mathrm{~mm} / \mathrm{s}$ ), and the starting inflexion (range 0.5 to $2 \mathrm{~mm}$ ). All the tests reported in Table 1 were repeated at least three times to assure process repeatability and reproducibility.

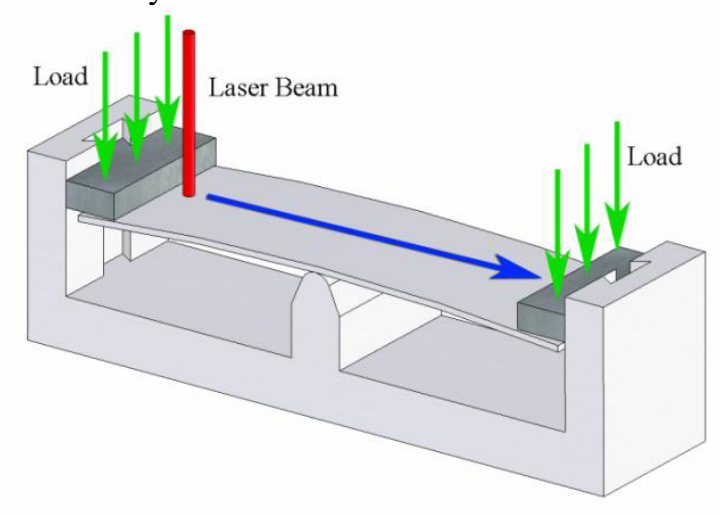

Figure 1. Diagram of the inflexion system used.

A full factorial design of experiment was employed to study the effects of laser treatment on the material behavior during the forming process. Table 1 summarizes the experimental plan developed. Three operational parameters were considered: laser power (range 500 to $800 \mathrm{~W}$ ), laser scan speed (range 4 to $7 \mathrm{~mm} / \mathrm{s}$ ), and the starting inflexion (range 0.5 to $2 \mathrm{~mm}$ ). All the tests reported in Table 1 were repeated at least three times to assure process repeatability and reproducibility. The outcome of the experimental plan was the residual inflection.

Table 1. Full factorial experimental plan.

\begin{tabular}{llll}
\hline \multicolumn{4}{c}{ Experimental Factors } \\
\hline $\begin{array}{c}\text { Experimental } \\
\text { Levels }\end{array}$ & $\begin{array}{c}\text { Laser } \\
\text { Power, W }\end{array}$ & $\begin{array}{c}\text { Laser speed, } \\
\mathbf{m m} / \mathbf{s}\end{array}$ & $\begin{array}{c}\text { Starting } \\
\text { Inflexion, } \\
\mathbf{m m}\end{array}$ \\
\hline$I$ & 500 & 4 & 0,5 \\
$I I$ & 600 & 5 & 1 \\
$I I I$ & 700 & 6 & 1,5 \\
$I V$ & 800 & 7 & 2 \\
\hline
\end{tabular}

\subsection{Uncertain Model of the process}

A standard linear regression model was developed, to link the process parameters (velocity v, laser Power P, and initial bending I) to the achieved residual bending $\mathrm{B}$ after the process. In addition, the total energy $E$ provided by the laser is considered also. Total energy is proportional to a term $P / v$. As a test case, the model described by a second order model following the equation:

$$
\begin{gathered}
B=a_{1}+a_{2} v+a_{3} P+a_{4} I+a_{5} E+a_{6} v P+a_{7} v I+a_{8} v E+a_{9} P I+ \\
+a_{10} P E+a_{11} I E+a_{12} v^{2}+a_{13} P^{2}+a_{14} I^{2}+a_{15} E^{2}
\end{gathered}
$$

A practical way to model the uncertainties inherent to the parameters of a model is to represent the model parameter by fuzzy numbers. Forming a special class of fuzzy sets, fuzzy numbers are characterized by a membership function $\mu(\mathrm{x}), 0<\mu(\mathrm{x})<1$, that defines the degree to which the parameter can take a certain value.

For the evaluation of systems with uncertain the Transformation Method, that is a practical 
implementation of the fuzzy arithmetic, can be used [9, 10]. The Transformation Method considers the model as a black box providing only an input-output relation that is a mapping between points in the input parameter hyperspace and points in the output space.

Among the advantages of the Transformation Method, its characteristic property of reducing the fuzzy arithmetic to multiple crisp-number operations entails that the Transformation Method can be implemented without major problems into an existing software environment for system simulation, avoiding expensive rewriting of the software. This approach was already used to describe the inherent uncertainties related to a model describing the EFB coating process [10] and FB finishing process [11]. The aim of this paper is to demonstrate the applicability of the method to the process of laser assisted bending, as well as its utility in the prediction of the residual bending variability and in operational parameters setting.

\section{Results and discussion}

The unknown coefficients $a_{1}, a_{2}, \ldots, a_{15}$ can be obtained from a linear regression algorithm using the experimental data. The dataset dispersion around this model is shown in Figure 2. It reports, for each parameter combination, the measured residual bending ( $x$ axis) and the corresponding model results ( $y$ axes).

The model is very rough in describing the physics of the process, thus it is essential to assess the inherent model uncertainty through the use of a fuzzy model that can be expressed as:

$$
\begin{gathered}
\quad \underline{B}=\underline{a}_{1}+\underline{a}_{2} v+\underline{a}_{3} P+\underline{a}_{4} I+\underline{a}_{5} E+\underline{a}_{6} v P+\underline{a}_{7} v I+\underline{a}_{8} v E+ \\
+\underline{a}_{9} P I+\underline{a}_{10} P E+\underline{a}_{11} I E+\underline{a}_{12} v+\underline{a}_{13} P^{2}+\underline{a}_{14} I^{2}+\underline{a}_{15} E^{2}
\end{gathered}
$$

where the regression coefficients $\underline{a}_{1}, \underline{a}_{2}, \ldots, \underline{a}_{15}$ are triangular fuzzy numbers. Each of them has the modal value coinciding with the results provided by the linear regression, while the support is appropriately chosen, based on the standard deviation provided by the linear regression analysis, so that all the experimental data are included at $\mu=0$.

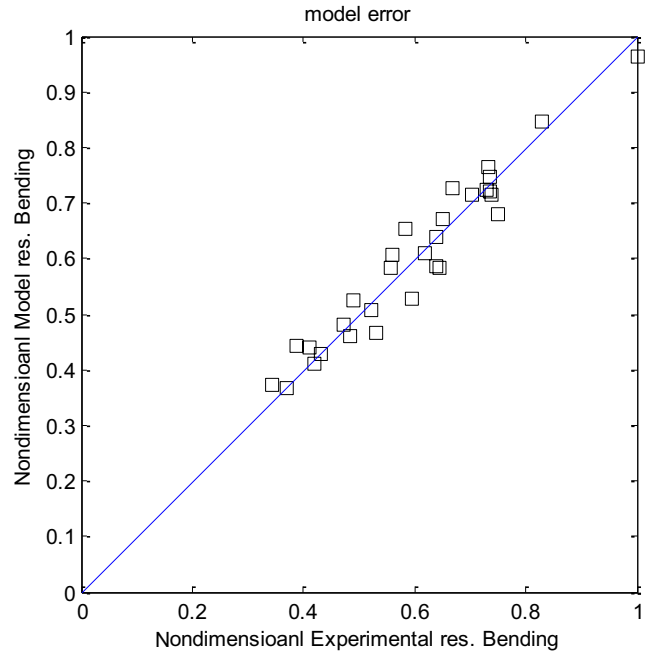

Figure 2. Dataset dispersion around the model. Blue line: model; red circles: experimental results

The fuzzy model can be used to evaluate how much a given experimental sample, characterized by $v_{i}, P_{i}, I_{i}, E_{i}$ and the corresponding bending $B_{i}$, belongs to the fuzzy set defined by the model. In particular, the crisp model does not represent any experimental data. As the level of uncertainty is increased, measured by a decrease in the membership function, the model accommodates a larger number of samples with lower membership level. The Transformation Method is used, given the input fuzzy numbers, to propagate to the residual bending the values of the input parameters: velocity $\mathrm{v}$, power $\mathrm{P}$, initial bending $\mathrm{I}$ and energy $\mathrm{E}$. The results are presented in Figure 3.

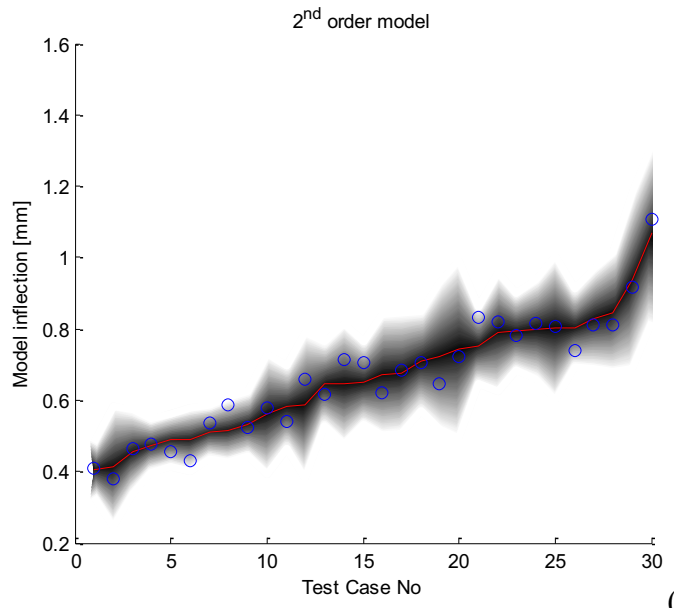

(a)

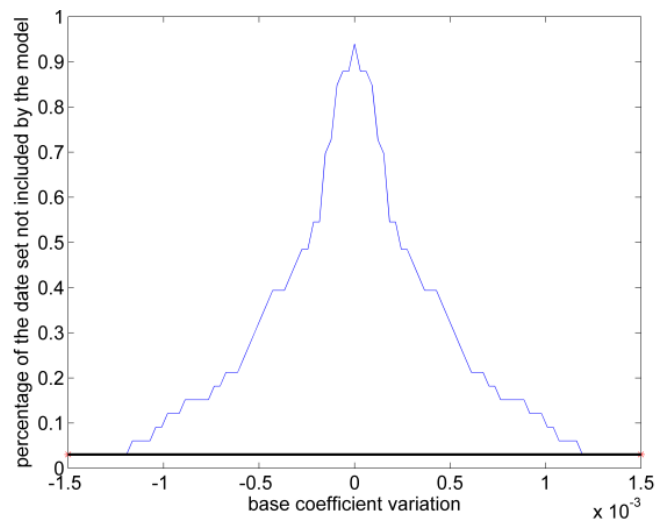

Figure 3. a) Results of the Transformation Method (grey shaded area), experimental results (blue); b) Percentage of experimental sample described by the model as a function of the membership level.

In Figure $3 \mathrm{a}$ the experimental data are represented as blue circles and the fuzzy model results are represented by the shaded area, where lighter zones refer to lower membership level. The graph in Figure $3 b$ suggests how much a given sample, characterized by a combination of process parameters and the corresponding residual bending, belongs to the fuzzy set. The fuzzy model can be used to predict the final bending of the sample, but it is also a useful tool to select operational parameters, as already shown in [8]. By assuming that a fixed bending must be obtained and after fixing a parameter e.g. the 
velocity. Therefore, for a desired value of the residual bending $B$, the inverse model provides the combinations of $P$ and $I$ leading to that result (intersection between the surface and the plane in Fig. 3a, red line on Fig. 3b). Moreover, it is possible to built a fuzzy process map where, as shaded areas, the uncertainty related to each operational point is reported. The fuzzy model produces a results that cannot be obtained from the crisp regression model that is: among all the points that produce the same desired residual bending, some of them (e.g. $I=1.5 \mathrm{~mm}$ and $P=510 \mathrm{~W})$ fall in areas where the model has a large uncertainty, while some of them (e.g. $I=0.5 \mathrm{~mm}$ $P=625 \mathrm{~W})$ fall in areas where the model is very precise.

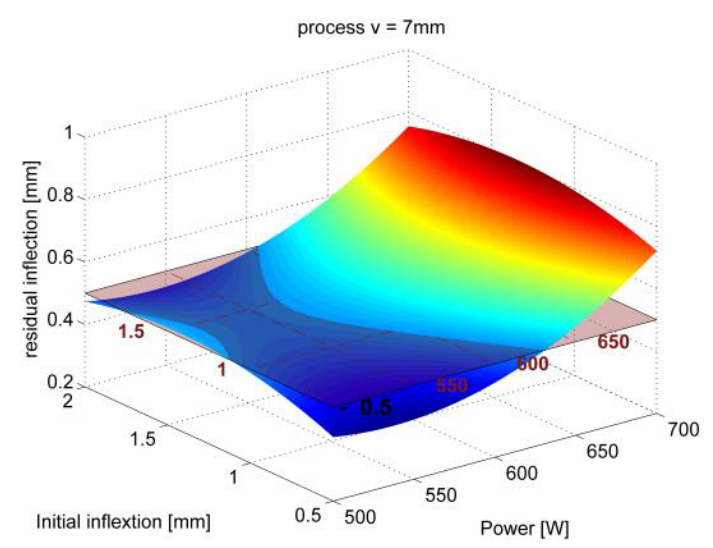

(a)

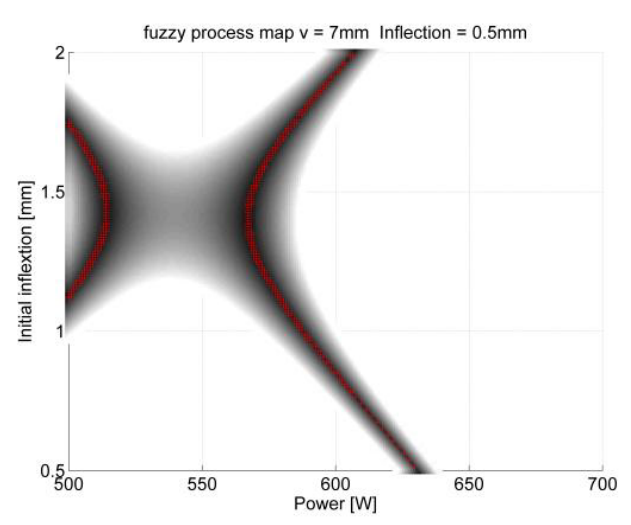

(b)

Figure 4. a) a) possible operational point from the crisp model b) Results of the Transformation Method (grey shaded area).

\section{Conclusions}

The fuzzy-logic based approach was used to describe the uncertainties of a model generated by an experimental investigation in the field of assisted laser bending. The fuzzy approach is used to evaluate how much a given sample, characterized by a combination of process parameters and the corresponding residual bending, belongs to the fuzzy set defined by the model. At the same time, the fuzzy model can be used to select process parameters and predict the inherent uncertainty related to the adopted model. This approach provides a tool to select, the model with the best fidelity, not just by looking a the "mean model" but also to the level of uncertainty that the model produces. Moreover, given a model and a set of parameters, the fuzzy approach allows the user to select the best operational point i.e. the one that is characterized by the lowest level of uncertainty.

\section{References}

1. Kalpakjian S, Schmid S. Manufacturing. Engineering and technology. 5th ed. 2005.

2. Tekiner Z. An experimental study on the examination of springback of sheet metals with several thicknesses and properties in bending dies. Journal of Materials Processing Technology, 145:109-17 (2004).

3. Vukota B. Sheet metal forming processes and die design. (2004)

4. Firat M, Kaftanoglu B, Eser O. Sheet metal forming analyses with an emphasis on the springback deformation. Journal of Materials Processing Technology, 196:135-48 (2008)

5. Eggertsen P-A, Mattiasson K. On the modelling of the bending-unbending behaviour for accurate springback predictions. International Journal of Mechanical Sciences, 51:547-63 (2009)

6. Liu YC. The effect of the restraining force on shape deviations in flanged channels. ASME Journal of Engineering Materials and Technology, 110: 389-94 (1988)

7. A. Gisario, M.Barletta, C.Conti, S.Guarino. Springback control in sheet metal bending by laserassisted bending: Experimental analysis, empirical and neural network modeling, Optics and Lasers in Engineering 49, 1372-1383 (2011).

8. M. Barletta, A Gisario, S. Guarino. Hybrid forming process of AA $6108 \mathrm{~T} 4$ thin sheets: modelling by neural network solutions. Proceedings of the Institution of Mechanical Engineers, Part B: Journal of Engineering Manufacture 223, 535-545 (2009).

9. O. Giannini M. Hanss M. "The component mode transformation method: A fast implementation of fuzzy arithmetic for uncertainty management in structural dynamics", Journal of Sound and Vibrations, 311, 1340-1357 (2011).

10. O. Giannini, M. Hanss, "An interdependency index for the outputs of uncertain systems", Fuzzy Sets and Systems 159, pp. 1292-1308, (2008)

11. F. Trovalusci, M. Barletta, O. Giannini, "Fuzzy model for electrostatic fluidized bed coating", Proceedings of the 12th Biennial Conference on Engineering Systems Design and Analysis ESDA14, June 25-27, 2014, Copenhagen, Denmark. 